\title{
Volatilização de amônia do solo após doses de ureia com inibidores de urease e de nitrificação na cultura do abacaxi
}

\author{
Deivisson Ferreira da Silva', Rodinei Facco Pegoraro ${ }^{2 *}$, Victor Martins Maia ${ }^{3}$, Marcos Koiti Kondo ${ }^{3}$, \\ Gleika Larisse Oliveira Dorasio de Souza ${ }^{3}$, Mauro Franco Castro Mota ${ }^{3}$
}

$10.1590 / 0034-737 X 201764030014$

\section{RESUMO}

Os inibidores de urease N-(n-butil) tiofosfóricotriamida (NBPT) e nitrificação dicianodiamida (DCD) minimizam as perdas de nitrogênio $(\mathrm{N})$ pela volatilização da amônia $\left(\mathrm{NH}_{3}\right)$, por redução da velocidade de hidrólise da ureia e conversão de amônio em nitrato, respectivamente. O objetivo deste estudo foi avaliar as perdas de $\mathrm{N}$ do solo por volatilização, após a adição de doses de $\mathrm{N}$, na forma de ureia combinada com inibidores de urease e de nitrificação, no semiárido mineiro. Utilizou-se o delineamento em blocos casualizados, com três repetições, em esquema fatorial 4 x 5 x 7 , constituídos por: quatro combinações de ureia com inibidores (ureia comum; ureia + NBPT; ureia + DCD e, ureia + NBPT + DCD), cinco doses de $\mathrm{N}(0,0 ; 3,7 ; 7,4 ; 11,1$ e 14,8 g/planta) e sete tempos de avaliação $(3,6,9,12,15,18$ e 21 dias $)$ após a aplicação, em cobertura, no cultivo de abacaxi irrigado. Foram avaliadas as perdas de amônia e o pH em torno do grânulo de ureia. $\mathrm{O}$ inibidor de urease (ureia + NBPT) foi mais eficiente nas menores doses de nitrogênio, além de reduzir e atrasar os períodos de volatilização de $\mathrm{N}_{-} \mathrm{NH}_{3}$ e também diminuir o $\mathrm{pH}$ do solo, por causa, provavelmente da maior nitrificação e, ou, do aumento da taxa de decomposição da matéria orgânica do solo (MOS), pela maior disponibilidade de $\mathrm{N}$ em comparação aos tratamentos com ureia e ureia + DCD. O aumento das doses de nitrogênio reduziu as perdas relativas de $\mathrm{N}$ por volatilização, graças à maior saturação dos sítios de ação da enzima urease.

Palavras-chave: nitrogênio; fertilizantes estabilizados; NBPT; DCD.

\section{ABSTRACT}

\section{Ammonia volatilization of soil after doses of urea with urease and nitrification inhibitors in pineapple culture}

The urease inhibitor N-(n-butyl) thiophosphorictriamide (NBPT) and nitrification inhibitor dicyandiamide (DCD) can minimize nitrogen $(\mathrm{N})$ losses by ammonia $\left(\mathrm{NH}_{3}\right)$ volatilization, decreasing the speed of urea hydrolysis and ammonium to nitrate conversion, respectively. The objective of this study was to evaluate the soil $\mathrm{N}$ losses by volatilization after the addition of $\mathrm{N}$ doses in the form of urea combined with urease and nitrification inhibitors in the Minas Gerais semiarid. A randomized complete block design was used with three replications and a $4 \times 5 \times 7$ factorial scheme - four combinations of urea inhibitors (common urea, urea + NBPT; urea + DCD; and urea + NBPT + DCD), five N levels ( 0.0 ; $3.7 ; 7.4 ; 11.1$; and $14.8 \mathrm{~g} / \mathrm{plant})$, and seven evaluation times $(3,6,9,12,15,18$, and 21 days $)$ - after topdressing in irrigated pineapple crop. The losses of ammonia and $\mathrm{pH}$ around the urea granule were evaluated. The urease inhibitor ( urea + NBPT) was more efficient in the lower N levels; in addition, it decreased and delayed the periods of $\mathrm{N}^{-\mathrm{NH}_{3}}$ volatilization and also decreased the $\mathrm{pH}$ of the soil, probably due to the higher nitrification and/or increase of the decomposition rate of soil organic matter by the greater availability of $\mathrm{N}$ compared with the urea and urea + DCD treatments. The relative $\mathrm{N}$ losses were reduced with the increase of $\mathrm{N}$ levels, because of the greater saturation of the enzyme urease action sites.

Key words: DCD; NBPT; nitrogen; stabilized fertilizers.

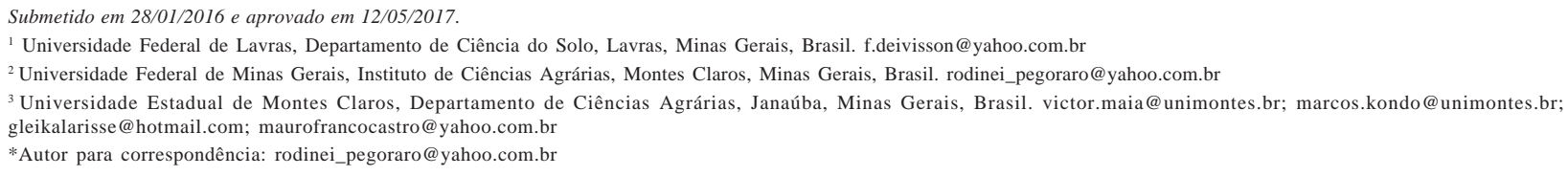




\section{INTRODUÇÃO}

A ureia tem sido a fonte nitrogenada mais utilizada, nas últimas décadas, em todo o mundo, por seu baixo custo e alta concentração de $\mathrm{N}(45 \%$ de N). Contudo, quando aplicada em superfície, as perdas por volatilização de amônia $\left(\mathrm{NH}_{3}\right)$ são elevadas, por causa da maior atividade da enzima urease. No Brasil, as perdas médias de $\mathrm{N}$ por volatilização são de 20 a 50\% do $\mathrm{N}$ aplicado (Trivelin et al., 2002; Cantarella et al., 2003, 2008), e ocorrem horas após a adubação com ureia, por causa de sua hidrólise enzimática (Da Ros et al., 2005), sendo a volatilização de $\mathrm{NH}_{3}$ aumentada pela presença de resíduos que ampliam a atividade da enzima urease no solo. A perda de $\mathrm{NH}_{3}$ depende da classe de solo, do $\mathrm{pH}$, da capacidade de tamponamento, da temperatura e da umidade (Tasca et al., 2011).

As perdas de $\mathrm{N}-\mathrm{NH}_{3}$ por volatilização têm importância econômica para os agricultores e afetam a qualidade da atmosfera e das águas subterrâneas (Zaman et al., 2008; Zaman et al.,2009; Zaman \& Blennerhassett, 2010). Recentemente, alguns compostos químicos, entre eles o NBPT (N-(n-butil) tiofosfóricotriamida), têm sido misturados à ureia com o objetivo de diminuir sua velocidade de hidrólise, por meio da inibição da atividade da urease (Tasca et al., 2011). Resultados experimentais mostram que esses aditivos diminuem a atividade da urease (SanzCobena et al., 2008) e, com isso, retardam a hidrólise da ureia e o pico de volatilização, diminuindo a quantidade de $\mathrm{NH}_{3}$ volatilizada (Sanz-Cobena et al., 2008). Além de inibidores de urease, existem no mercado os inibidores de nitrificação, como a dicianodiamida (DCD), um composto desenvolvido para reduzir as perdas de $\mathrm{N}$ por lixiviação, diminuindo a formação de $\mathrm{NO}_{3}{ }^{-}$e preservando por mais tempo no solo a forma menos lixiviável, a de $\mathrm{NH}_{4}^{+}$(Trenkel, 2010).

A utilização de inibidores de urease e de nitrificação, combinada com irrigação, em regiões em que a temperatura média anual é elevada, reduz as perdas de $\mathrm{N}$ do solo. No entanto, são escassos os estudos sobre perdas por volatilização após a adição de fertilizantes nitrogenados, com inibidores de urease e de nitrificação, em sistemas irrigados no semiárido. Diante do exposto, objetivou-se avaliar as perdas de $\mathrm{N}$ do solo por volatilização após a adição de doses de $\mathrm{N}$, na forma de ureia combinada com inibidores de urease e de nitrificação, no semiárido mineiro.

\section{MATERIAL E MÉTODOS}

O estudo foi desenvolvido no município de Janaúba, MG, situado a $15^{\circ} 47^{\prime} \mathrm{S}$ e $43^{\circ} 18^{\prime} \mathrm{O}$, com $516 \mathrm{~m}$ de altitude. O clima da região é o Aw, segundo a classificação de Köppen, ou seja, clima tropical, com inverno seco e preci- pitação pluviométrica média de $870 \mathrm{~mm}$, distribuída irregularmente no período chuvoso de outubro a março, com temperatura média anual de $25^{\circ} \mathrm{C}$, insolação de $2.700 \mathrm{~h}$ anuais e umidade do ar de 65\%,em média (INMET, 2015).

Antes do estudo, foram coletadas dez subamostras de solo nas camadas de $0-20 \mathrm{~cm}$ de profundidade, distribuídas aleatoriamente na área, para avaliação dos atributos químicos e físicos do solo, conforme Embrapa (1997). O solo da área experimental foi classificado como Latossolo Vermelho eutrófico, de textura média (Embrapa, 2013). A análise do solo na camada de 0-20 cm evidenciou as seguintes características: 259, 187 e $554 \mathrm{~g} \mathrm{~kg}^{-1}$ de argila, silte e areia, respectivamente; $2,19 \mathrm{dag} \mathrm{kg}^{-1}$ de matéria orgânica; 5,7 de $\mathrm{pH}\left(\mathrm{H}_{2} \mathrm{O}\right) ; 2,60 \mathrm{mg} \mathrm{dm}^{-3} \mathrm{de} \mathrm{P}$ (Mehlich 1); $133,6 \mathrm{mg} \mathrm{dm}^{-3} \mathrm{de} \mathrm{K}$ (Mehlich 1); $2,1 \mathrm{mg} \mathrm{dm}^{-3} \mathrm{de} S ; 1,3 \mathrm{cmol}_{\text {c }}$ $\mathrm{dm}^{-3}$ de $\mathrm{Ca} ; 0,4 \mathrm{cmol}_{\mathrm{c}} \mathrm{dm}^{-3}$ de $\mathrm{Mg} ; 0,26 \mathrm{cmol}_{\mathrm{c}} \mathrm{dm}^{-3}$ de $\mathrm{Al}$ (extrator $\mathrm{KCl} 1,0 \mathrm{~mol} \mathrm{~L}^{-1}$ ) e 2,0 $\mathrm{cmol}_{c} \mathrm{dm}^{-3} \mathrm{de} \mathrm{H}+\mathrm{Al}$ (extrator acetato de cálcio $0,5 \mathrm{~mol} \mathrm{~L}^{-1}$ a pH 7,0).

Aárea foi cultivada com abacaxizeiro 'Vitória', plantado em agosto de 2012, com mudas do tipo filhote, previamente selecionadas pelo tamanho. $\mathrm{O}$ espaçamento para fileiras duplas foi de $0,9 \times 0,40 \times 0,20 \mathrm{~m}$ (totalizando 76.923 plantas $h^{-1}$ ). A adubação de plantio foi realizada com 16,7 g/planta de superfosfato simples, distribuído nos sulcos, e a adubação de cobertura, com $\mathrm{K}$ e micronutrientes, foi parcelada em seis vezes, totalizando $75 \mathrm{~g} /$ planta de cloreto de potássio, no período vegetativo do abacaxizeiro. Os micronutrientes foram aplicados por via foliar com ácido bórico $(0,07 \%)$, sulfato de zinco $(0,1 \%)$ e sulfato de cobre $(0,1 \%)$, no mesmo período da adubação feita com $\mathrm{K}$ em cobertura. A cultura foi irrigada por aspersão convencional fixa, com miniaspersores de vazão nominal de $200 \mathrm{~L} \mathrm{~h}^{-1}$. Os emissores foram espaçados por $4 \mathrm{~m}$ ao longo da linha e 3 $\mathrm{m}$ entre as linhas. Foram realizadas três irrigações semanais, sendo os tempos de irrigação calculados a partir da soma das evapotranspirações de referência $\left(\mathrm{ET}_{0}\right)$, obtidas pelo método de Hargreaves-Samani, multiplicadas pelos coeficientes da cultura $\left(\mathrm{K}_{\mathrm{c}}\right)(0,5-0,7)$, divididos pela eficiência do sistema de irrigação bem como pela intensidade de aplicação $\left(I_{a}\right)$.

A avaliação das perdas de $\mathrm{N}$ por volatilização iniciouse em outubro de 2014, durante o desenvolvimento vegetativo do abacaxizeiro. Para tanto, adotou-se o delineamento em blocos casualizados, com quatro repetições, sendo a unidade experimental arranjada em esquema fatorial 4 × 5 × 7, com quatro combinações de ureia e inibidores (1- ureia comum; 2- ureia + inibidor de urease; 3- ureia + inibidor de nitrificação; 4- ureia + inibidores de urease e de nitrificação), cinco doses de $\mathrm{N}(0,0 ; 3,7 ; 7,4$; 11,1 e 14,8 g/planta) e sete tempos de avaliação aos 3, 6, 9, $12,15,18$ e 21 dias após a aplicação dos tratamentos.

Os dados climáticos de temperatura (máxima, mínima e média), radiação, velocidade do vento e umidade relativa 
foram mensurados diariamente, em estação meteorológica instalada a 150 metros da área experimental (Figura 1).

As perdas de $\mathrm{NH}_{3}$ para a atmosfera foram mensuradas por meio de câmaras estáticas e semiabertas, colocadas sobre bases feitas com PVC, com 0,20 m de diâmetro e 0,07 $\mathrm{m}$ de altura. As bases foram cravadas nas entrelinhas das fileiras duplas dos abacaxizeiros, deixando aproximadamente 0,04 m do tubo acima da superfície do solo.

As câmaras foram construídas conforme o modelo desenvolvido por Nommik (1973), modificado por Cantarella et al. (2003), em que, em vez de uma base para cada câmara, foram utilizadas cinco bases, sobre as quais a câmara foi rotacionada em intervalos de três dias. As câmaras consistiram em tubos de PVC com 0,20 m de diâmetro e 0,40 m de altura. Sobre cada tubo, foi colocado um suporte de metal, ao qual acoplou-se uma tampa plástica protetora, com espaço de 0,01 m para passagem de ar, impedindo a entrada de umidade e de raios solares que pudessem afetar o sistema internamente.

Em cada câmara coletora, foram colocados dois discos de espuma de polietileno com 0,02 m de espessura, embebidos em $100 \mathrm{~mL}$ de uma solução preparada com 50 $\mathrm{mL}$ de $\mathrm{H}_{3} \mathrm{PO}_{4}$ e $40 \mathrm{~mL}$ de glicerina por litro e, em seguida, foram levemente comprimidos para que atingissem um volume final de solução de $70 \mathrm{~mL}$, evitando o gotejamento no interior dos cilindros. $\mathrm{O}$ primeiro disco, colocado a $0,15 \mathrm{~m}$ do solo, absorveu o $\mathrm{NH}_{3}$ volatilizado. O segundo disco, a 0,30 m de altura, teve a função de captar a amônia proveniente da atmosfera, impedindo a contaminação do disco inferior.

O inibidor de urease utilizado nos tratamentos com ureia foi o UREMAX NBPT ${ }^{\circledR}$, na dose de 6,5 g para cada $1.000 \mathrm{~g}$ de nitrogênio (Adfert, 2015). O inibidor de nitrificação foi a DCD, misturada com ureia na dose de 100 g para cada $1.000 \mathrm{~g}$ de nitrogênio (Soares et al., 2012). O nitrogênio presente na DCD também foi considerado para a estimativa das doses equivalentes de $\mathrm{N}$ nos tratamentos. A ureia e as misturas com inibidores foram pesadas em balança de precisão e dispostas na superfície do solo em faixas, sem incorporação, localizadas no interior das bases (das câmaras estáticas).

As perdas de amônia foram avaliadas aos 3, 6, 9, 12, 15, 18 e 21 dias após a aplicação dos tratamentos no solo. Após cada coleta, o fosfato de amônio formado foi extraído imediatamente dos discos de espuma, por meio de oito lavagens sucessivas com solução de $\mathrm{KCl} 1 \mathrm{~mol} \mathrm{~L}^{-1}$. O produto dessa lavagem foi coletado em balões volumétricos, e o volume ajustado para $500 \mathrm{~mL}$ com a solução de $\mathrm{KCl} 1 \mathrm{~mol} \mathrm{~L}^{-1}$, retirando-se alíquota de $20 \mathrm{~mL}$ para determinação do equivalente em teor de $\mathrm{N}-\mathrm{NH}_{3}$, em destilador de arraste de vapor semimicro Kjeldahl, segundo metodologia descrita por Tedesco et al. (1995). O cálculo do nitrogênio amoniacal baseou-se nos volumes de ácido clorídrico gasto na titulação das provas em branco e das amostras, de acordo com a seguinte equação:

$\mathrm{N}-\mathrm{NH}_{3}=\left[\frac{(\text { Vac }- \text { Vbr) 14,007 Nac }}{\text { Val }}\right] \mathrm{Vt}$, em que:

$\mathrm{N}-\mathrm{NH}_{3}=$ Nitrogênio amoniacal;

Vac $=$ Volume de ácido clorídrico gasto na titulação da $\operatorname{amostra}(\mathrm{mL})$;

Vbr = Volume do ácido clorídrico gasto na titulação do branco de análise $(\mathrm{mL})$;

14,007 = Constante equivalente a massa atômica do N;

$\mathrm{Nac}=$ Normalidade do ácido clorídrico utilizado;

Val = Volume da alíquota usada na destilação $(\mathrm{mL})$;

$\mathrm{Vt}=$ Volume total de extrato utilizado no processo de extração $(\mathrm{mL})$.

Foram coletadas amostras de solo, antes de cada avaliação de perdas de $\mathrm{NH}_{3}$, na profundidade de $0-20 \mathrm{~cm}$, para
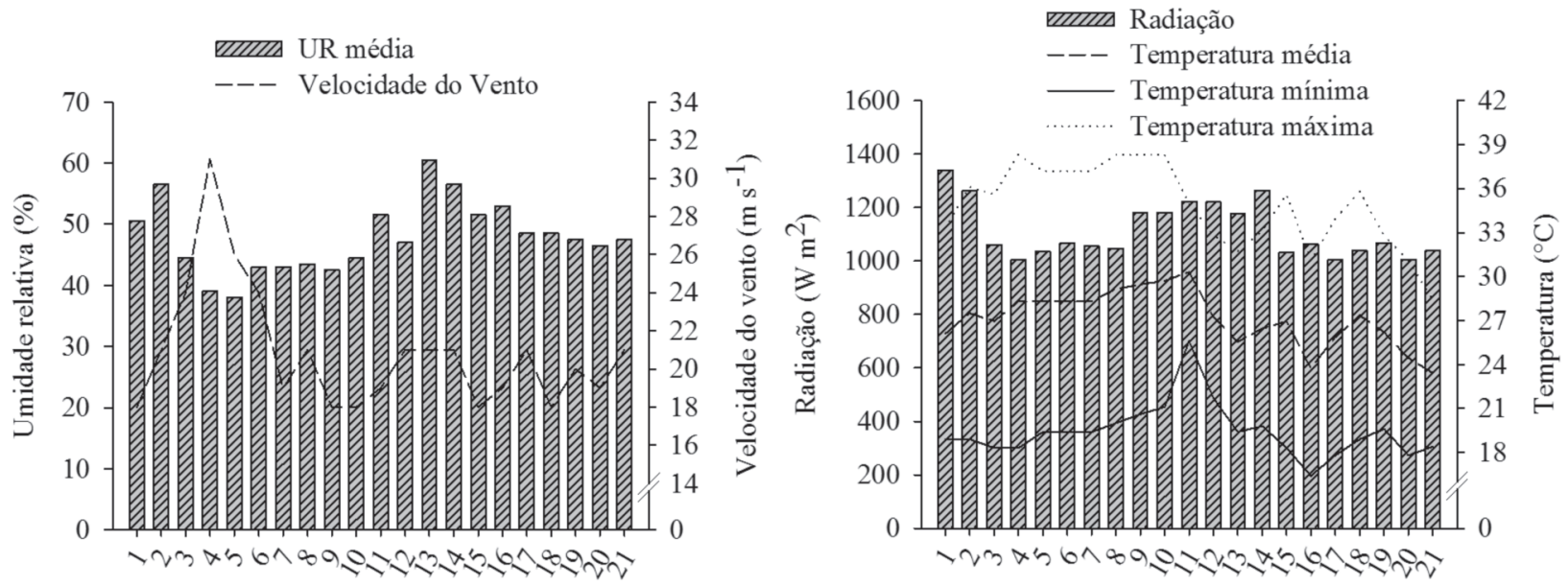

Figura 1: Médias diárias de umidade relativa (UR), velocidade do vento, radiação e temperaturas (mínima, máxima e média), durante o desenvolvimento do estudo. 
avaliação do $\mathrm{pH}$ do solo, localizado nas proximidades do grânulo da ureia, como indicativo da atividade da enzima urease.

A análise estatística dos dados incluiu análises de variância, teste de Tukey e regressão, a 5\% de significância, por meio do software Sisvar (Ferreira, 2011). Os modelos foram ajustados com base na significância dos parâmetros e no coeficiente de determinação.

\section{RESULTADOS E DISCUSSÃO}

De acordo com o resultado obtido após análise de variância, os valores de $\mathrm{pH}_{(\mathrm{H} 2 \mathrm{O})}$ da camada de $0-2 \mathrm{~cm}$ do solo foram influenciados $(\mathrm{p}<0,05)$ pela interação dupla entre os fatores doses de $\mathrm{N}$ versus combinações de inibidores e entre doses de $\mathrm{N}$ versus tempo, além de todos os demais fatores isolados (Tabela 1).

$\mathrm{O}$ valor de $\mathrm{pH}$ do solo foi menor no tratamento com ureia + NBPT e, maior quando se utilizou ureia + NBPT + DCD (Tabela 2) o que pode estar relacionado com o processo de nitrificação mais intenso, em virtude, possivelmente, dos teores de amônio no solo, maiores que os dos demais tratamentos nos quais as perdas de amônia foram mais elevadas. A maior disponibilidade de N, no solo do tratamento com ureia + NBPT, também pode ter ocasionado aumento da taxa de decomposição da MOS, diminuindo o $\mathrm{pH}$ do solo. A maior presença de $\mathrm{N}$ no solo promove a atividade microbiana responsável pela decomposição da MOS que pode aumentar a concentração de ácido carbônico $\left(\mathrm{CO}_{2}+\mathrm{H}_{2} \mathrm{O} \longleftrightarrow \mathrm{H}_{2} \mathrm{CO}_{3}\right)$, que se dissocia $\left(\mathrm{H}_{2} \mathrm{CO}_{3}\right.$ $\longleftrightarrow \mathrm{HCO}_{3}+\mathrm{H}^{+}$) e libera íons $\mathrm{H}^{+}$, reduzindo o $\mathrm{pH}$ do solo (Reis et al., 2016).

A presença da DCD no tratamento com inibidor de urease mais nitrificação (ureia + NBPT + DCD) impediu a transformação do amônio em nitrato, limitando a reação de nitrificação, pela qual se produzem dois moles de $\mathrm{H}^{+}$ para cada mol de $\mathrm{NH}_{4}^{+}$oxidado, o que manteve o $\mathrm{pH}$ elevado em virtude do processo de hidrólise da ureia, que é responsável pelo aumento do $\mathrm{pH}$. A inibição da nitrificação propiciou maior $\mathrm{pH}$ do solo em torno do grânulo da ureia, do que no tratamento com ureia + NBPT, em que o processo de nitrificação contribuiu para redução do pH. A DCD, controlando a nitrificação, mantém o pH do solo elevado por mais tempo, associado à maior concentração de $\mathrm{NH}_{4}^{+}$ causada pela hidrólise da ureia (Soares et al., 2012).

Independendemente das combinações de ureia com inibidores, os valores de $\mathrm{pH}$ apresentaram comportamento sigmoidal em função das doses de nitrogênio. Os valores máximos ocorreram com doses de 2,10; 0,33, 0,39 e 0,46 g/planta, respectivamente, para ureia, ureia + NBPT, ureia + DCD e ureia + NBPT + DCD (Figura 2). A maior dose requerida no tratamento com ureia para atingir o valor máximo de $\mathrm{pH}$ foi relacionada com a ausência dos inibidores, principalmente o NBPT, que atua nos sítios de ação da enzima urease, impedindo a hidrólise da ureia. A estabilização da curva a partir desses pontos indica a saturação dos sítios ativos dessa enzima. Longo \& Melo (2005), avaliando a velocidade de hidrólise da ureia em

Tabela 2: Médias de $\mathrm{pH}_{(\mathrm{H} 2 \mathrm{O})}$ do solo coletado na camada de 0-2 $\mathrm{cm}$ do entorno do grânulo de ureia, após a adição de diferentes doses de nitrogênio e de distintas combinações de ureia com inibidores de urease e de nitrificação: ureia (U), N-(n-butil) tiofosfórico triamida (NBPT), Dicianodiamida (DCD)

\begin{tabular}{ll}
\hline Combinações & \multicolumn{1}{c}{$\mathbf{p H}$} \\
\hline U & $7,80 \mathrm{ab}^{*}$ \\
U + NBPT & $7,78 \mathrm{~b}$ \\
U + DCD & $7,87 \mathrm{ab}$ \\
U + NBPT + DCD & $7,89 \mathrm{a}$ \\
Média geral & 7,84 \\
\hline
\end{tabular}

*Médias seguidas por mesma letra na coluna não diferem entre si pelo teste de Tukey a 5\% de significância.

Tabela 1: Resumo da análise de variância para $\mathrm{pH}_{(\mathrm{H} 2 \mathrm{O})}$ na camada de $0-2 \mathrm{~cm}$ de solo, $\mathrm{N}_{-} \mathrm{NH}_{3}$ perdido e N-NH $\mathrm{N}_{3}$ acumulado, após a adição de diferentes doses de N (D), distintas combinações de ureia com inibidores de urease (NBPT) e de nitrificação (Dicianodiamida), para avaliação no tempo (T)

\begin{tabular}{|c|c|c|c|c|}
\hline \multirow{2}{*}{ Fonte de variação } & \multirow{2}{*}{ GL } & \multicolumn{3}{|c|}{ Quadrado Médio } \\
\hline & & $\mathrm{pH}$ & $\mathrm{N}-\mathrm{NH}_{3}$ perdido $\left(\mathrm{kg} \mathrm{ha}^{-1}\right)$ & $\mathrm{N}-\mathrm{NH}_{3}$ acumulado $(\%)$ \\
\hline Bloco & 3 & $0,11^{\mathrm{ns}}$ & $546,63 *$ & $630,59^{\mathrm{ns}}$ \\
\hline Dose de N (D) & 4 & $14,07 * *$ & $23.924,38 * *$ & $10.983,81 * *$ \\
\hline Combinações (F) & 3 & $0,32 * *$ & $4.520,24 * *$ & $31.822,09 * *$ \\
\hline Tempo $(\mathrm{T})$ & 6 & $9,79 * *$ & $20.911,58 * *$ & $25.960,87 * *$ \\
\hline$D$ versus $F$ & 12 & $0,26 * *$ & $769,65 * *$ & $1.481,53 * *$ \\
\hline D versus $\mathrm{T}$ & 24 & $0,95 * *$ & $2.204,02 * *$ & $28,58^{\mathrm{ns}}$ \\
\hline $\mathrm{F}$ versus $\mathrm{T}$ & 18 & $0,04^{\mathrm{ns}}$ & $5.396,91 * *$ & $725,88^{\mathrm{ns}}$ \\
\hline D versus $\mathrm{F}$ versus $\mathrm{T}$ & 72 & $0,10^{\mathrm{ns}}$ & $576,33 * *$ & $886,18^{\mathrm{ns}}$ \\
\hline Resíduo & 278 & 0,09 & 120,97 & 655,94 \\
\hline $\mathrm{CV}(\%)$ & & 3,99 & 44,13 & 21,01 \\
\hline Média geral & & 7,84 & 24,84 & 25,85 \\
\hline
\end{tabular}

*,**, ns Significativo a 5 e $1 \%$, e não significativo pelo teste $\mathrm{F}$.

Rev. Ceres, Viçosa, v. 64, n.3, p. 327-335, mai/jun, 2017 
diferentes concentrações, com doses variando de 1 até 6 $\mathrm{g} \mathrm{dm}^{-3}$, em diferentes latossolos, observaram que a concentração de $2,5 \mathrm{~g} \mathrm{dm}^{-3}$ foi suficiente para a saturação dos sítios da enzima no Latossolo Vermelho distrófico.

Para a interação doses versus tempo foi ajustado o modelo Gaussiano, observando-se o máximo valor de $\mathrm{pH}$, de 8,60 , com dose de 9,45 g/planta de N, no $12^{\circ}$ dia após a aplicação das combinações de ureia com os inibidores (Figura 3). Dessa forma, indica-se o ponto máximo de velocidade de hidrólise da ureia no solo e, também, o possível decréscimo da atividade da enzima urease.

De acordo com os resultados obtidos após análise de variância, as perdas por volatilização de $\mathrm{N}_{-} \mathrm{NH}_{3}$ foram influenciadas $(\mathrm{p}<0,05)$ pelos fatores: doses de $\mathrm{N}$ versus combinações de ureia e inibidores versus tempo, indicando interação tripla entre os fatores estudados (Tabela 1).

As perdas do $\mathrm{N}_{-} \mathrm{NH}_{3}$ variaram com a aplicação das diferentes combinações nitrogenadas, doses e tempo após

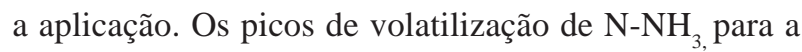
fonte ureia e a combinação ureia + DCD, foram similares; com ureia, o pico foi de $113,9 \mathrm{~kg} \mathrm{ha}^{-1}(12,47 \%$ do $\mathrm{N}$ aplicado), aos 5,25 dias após a aplicação, com dose de 11,76 g/ planta de nitrogênio; para ureia + DCD, foi de $113,49 \mathrm{~kg}$ ha $^{-1}$ (12,42\% do N aplicado) aos 5,25 dias, com a aplicação de 11,86 g/planta de N (Figura 4).

A utilização de NBPT propiciou redução e atraso dos picos de volatilização de $\mathrm{N}-\mathrm{NH}_{3}$. No tratamento com ureia + NBPT, o pico de volatilização de $\mathrm{N}^{-\mathrm{NH}_{3}}$ foi de $83,18 \mathrm{~kg}$
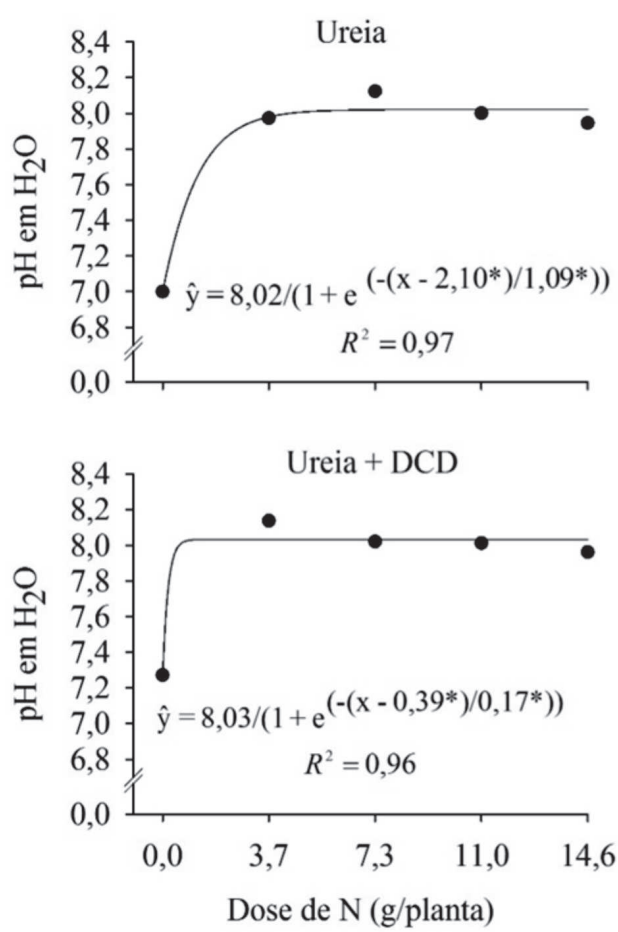

ha $^{-1}$ (8,45\% do $\mathrm{N}$ aplicado) e ocorreu 9,18 dias após a aplicação, com dose de 13,79 g/planta. Para a combinação ureia + NBPT + DCD, as perdas de $\mathrm{N}-\mathrm{NH}_{3}$ foram de 84,54 $\mathrm{kg} \mathrm{ha}^{-1}$ (8,59\% do $\mathrm{N}$ aplicado), aos 10,5 dias, com dose de 13,02 g/planta (Figura 4), e no tratamento com ureia, o pico de volatilização de $\mathrm{N}-\mathrm{NH}_{3}$ foi de $115,06 \mathrm{~kg} \mathrm{ha}^{-1}$ e ocorreu 5,79 dias após a aplicação, com dose de 11,76 g/planta.

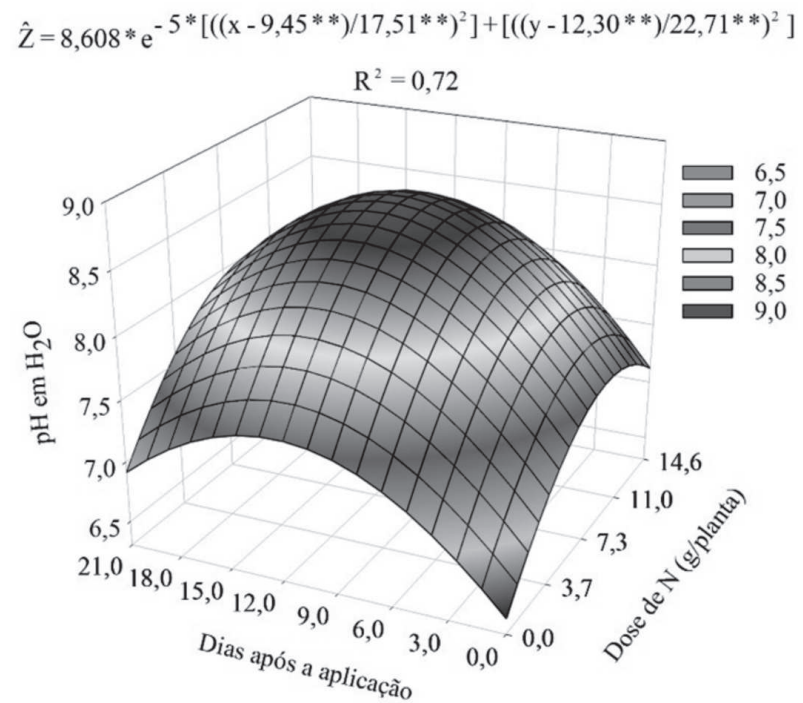

Figura 3: Valores de $\mathrm{pH}$ em amostras de solo coletadas em torno do grânulo de ureia (0-2 cm), após a adição de diferentes doses de nitrogênio e dias após adubação.," * : Significativo a 5 e $1 \%$ pelo teste F.
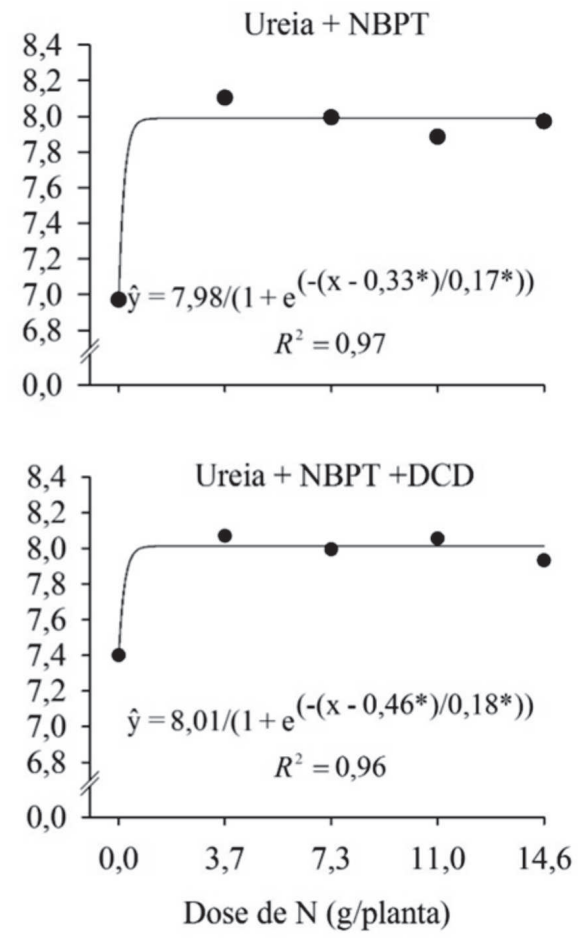

Figura 2: Valores de pH em amostras de solo coletadas em torno do grânulo de ureia $(0-2 \mathrm{~cm})$, após a adição de diferentes doses de nitrogênio e inibidores de uréase e de nitrificação [ureia (U); N-(n-butil) tiofosfórico triamida (NBPT); Dicianodiamida (DCD)]. * **: Significativo a 5 e $1 \%$ pelo teste $\mathrm{F}$. 
Vários estudos têm relatado a eficácia do NBPT adicionado à ureia em reduzir e retardar as perdas por volatilização de amônia (Zaman et al., 2009; Dawar et al., 2011; Sanz-Cobena et al., 2011). Em ambiente controlado, observa-se retardamento e redução das perdas por volatilização do $\mathrm{N}-\mathrm{NH}_{3}$, variando de sete a nove dias após a aplicação da ureia + NBPT (Soares et al., 2012).

A redução da hidrólise da ureia em virtude da adição de NBPT mantém o N-fertilizante na forma amídica (N$\mathrm{NH}_{2}$ ) por mais tempo, graças à redução da atividade da enzima urease. Assim, o inibidor evita a alta concentração da forma amoniacal $\mathrm{NH}_{4}^{+}$e o $\mathrm{pH}$ elevado na zona de aplicação do fertilizante, diminuindo a volatilização de $\mathrm{NH}_{3}$ (Zaman et al., 2009; Dawar et al., 2011; Grohs et al., 2011; Stafanato et al., 2013).
Com a utilização do inibidor de urease foram necessárias maiores quantidades de ureia $(\mathrm{N})$ para atingir o pico máximo de volatilização. Este efeito foi relacionado com a redução da hidrólise da ureia.

A volatização do $\mathrm{N}-\mathrm{NH}_{3}$ acumulado foi influenciada ( $\mathrm{p}$ $<0,05)$ pela interação dupla entre doses de $\mathrm{N}$ versus combinações de ureia com inibidores, além de todos os demais fatores isolados (Tabela 1).

A adição do inibidor de urease (NBPT) reduziu as perdas de $\mathrm{N}$ em relação à ureia aplicada em forma única e com inibidor de nitrificação (DCD), exceto na dose 14,8 g/planta (Figura 5). As reduções de perdas de $\mathrm{N}$ por volatilização na presença de NBPT foram de: a) 24,2\%, após a aplicação de $3,7 \mathrm{~g} /$ planta de N, no tratamento ureia + NBPT + DCD; b) 22,8\%, após a aplicação de 7,4 g/planta de N, no
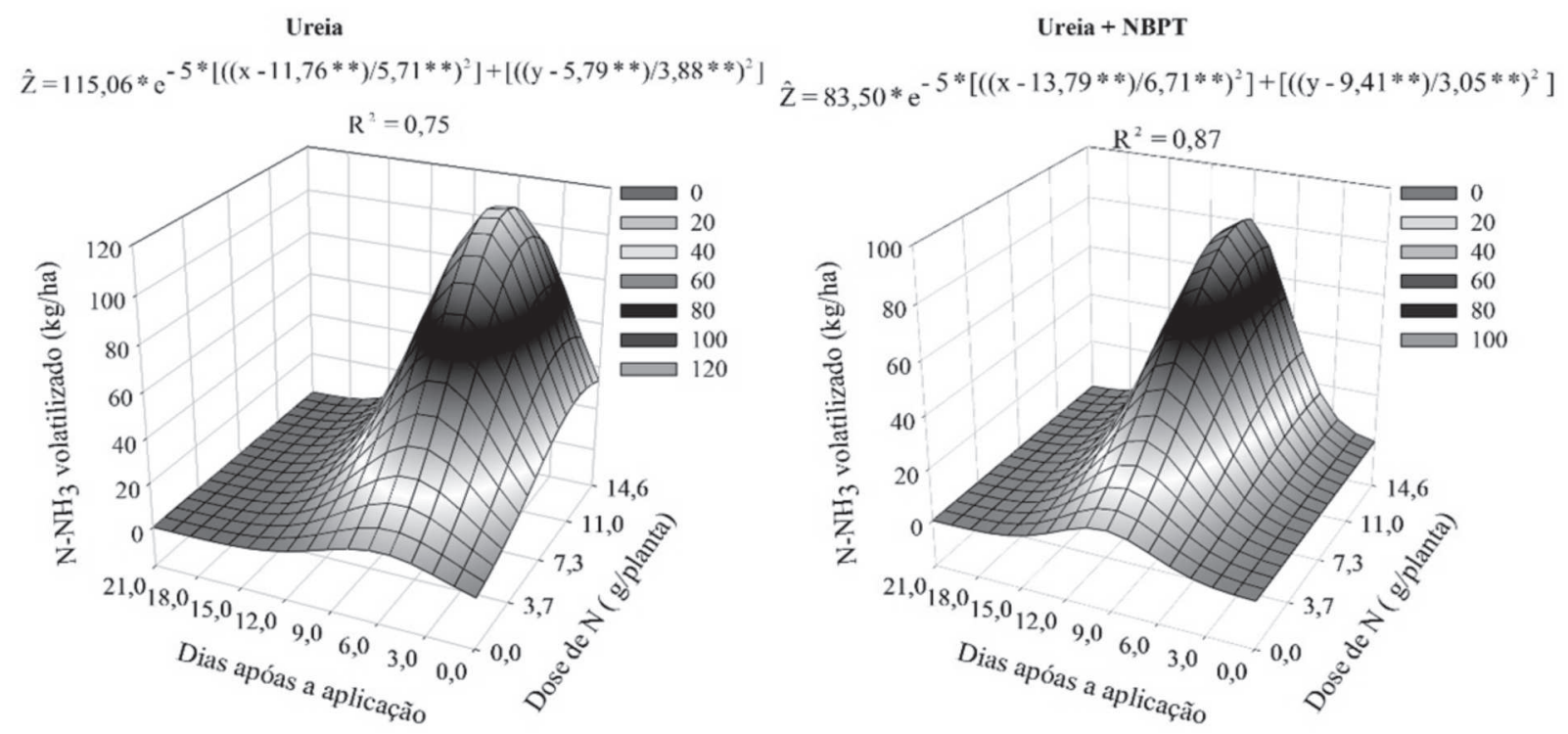

Ureia + DCD

$\hat{\mathrm{Z}}=115,36 * \mathrm{e}^{-5^{*}\left[\left((\mathrm{x}-11,84 * *) / 6,01^{* *}\right)^{2}\right]+\left[((\mathrm{y}-5,89 * *) / 3,54 * *)^{2}\right]}$
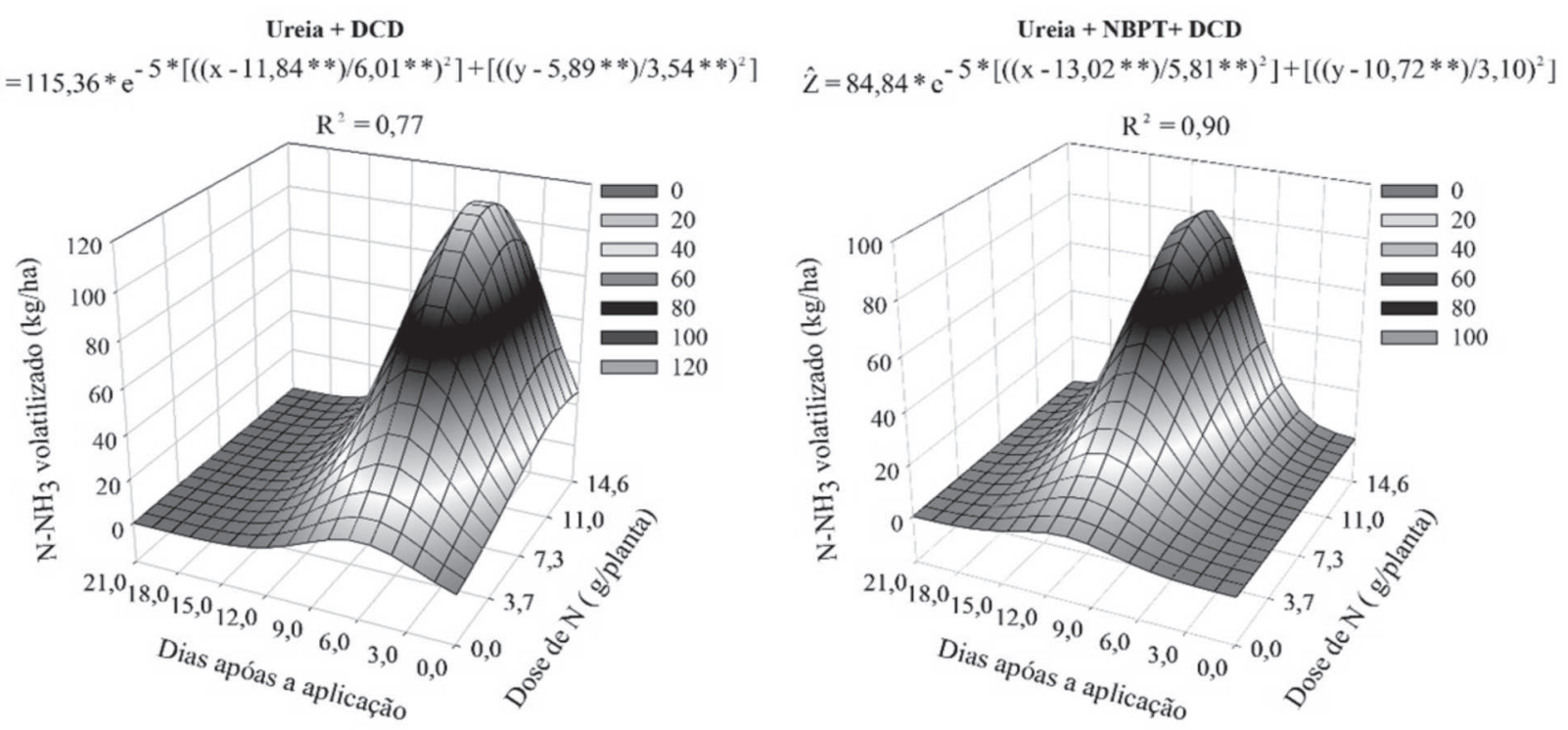

Figura 4: Perdas de N-NH 3 por volatilização durante 21 dias após a adubação com doses de nitrogênio e para as distintas combinações de ureia com inibidores de urease e de nitrificação [ureia (U); N-(n-butil) tiofosfórico triamida (NBPT); Dicianodiamida (DCD)]. , * : Significativo a 5 e $1 \%$ pelo teste $\mathrm{F}$.

Rev. Ceres, Viçosa, v. 64, n.3, p. 327-335, mai/jun, 2017 
tratamento ureia + NBPT + DCD; c) 17,9\%, após a aplicação de 11,1 g/planta de N, no tratamento ureia + NBPT.

A adição do inibidor de urease (NBPT) reduziu a hidrólise da ureia, o que diminuiu a volatilização do $\mathrm{N}$ $\mathrm{NH}_{3}$ (Figura 5). Em contrapartida, as maiores perdas de $\mathrm{N}$ $\mathrm{NH}_{3}$ observadas no tratamento com ureia e ureia + DCD foram atribuídas ao rápido aumento dos teores de $\mathrm{N}-\mathrm{NH}_{4}^{+}$ no solo, após a adubação, o que intensifica o processo de perda do N por volatilização. A adição de DCD inibiu o processo de nitrificação, retardando a oxidação do $\mathrm{N}_{-} \mathrm{NH}_{4}^{+}$ a $\mathrm{N}^{-\mathrm{NO}_{3}}$. Nesta condição, a maior concentração de N$\mathrm{NH}_{4}^{+}$próxima dos sítios de atividade da urease aumentou o $\mathrm{pH}$ e as perdas de $\mathrm{N}-\mathrm{NH}_{3}$.

$\mathrm{O}$ aumento das perdas por volatilização de $\mathrm{NH}_{3}$, pelo maior acúmulo de $\mathrm{N}_{-} \mathrm{NH}_{4}{ }^{+}$no solo, em virtude da adição de DCD, foi observado por Gioacchini et al. (2002) e por Zaman et al. (2008), mas, em ambos os casos, por causa das condições climáticas, as perdas de $\mathrm{N}$ foram mais baixas que as observadas neste estudo, sendo de 4,0 a 9,6\% e 4,2 a 5,7\% do N aplicado, respectivamente. Vários estudos têm relatado as perdas elevadas de $\mathrm{N}-\mathrm{NH}_{3}$, de até $60 \%$ do $\mathrm{N}$ aplicado, com média variando entre 20 e $30 \%$, para experimentos em condições de campo (Lara Cabezas et al., 2000; Trivelin et al., 2002; Cantarella et al., 2003; Cantarella et al., 2008).
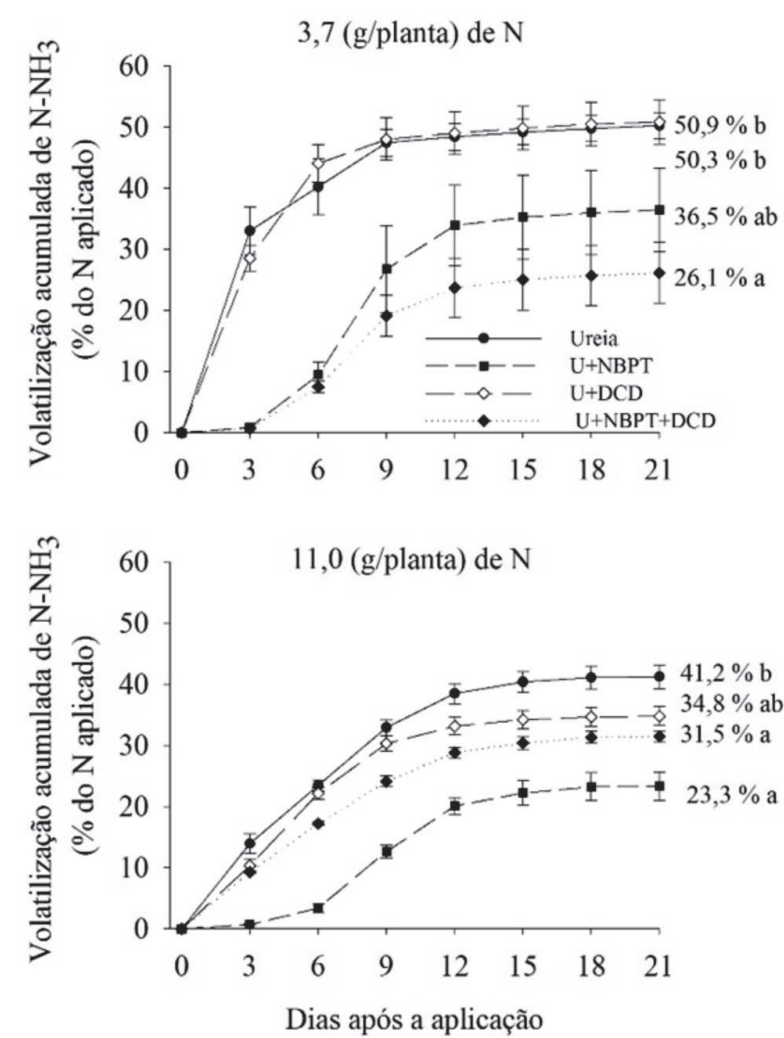

Figura 5: Perdas acumuladas de $\mathrm{N}-\mathrm{NH}_{3}$ durante 21 dias após a adição de diferentes doses de nitrogênio e para as distintas combinações de ureia com inibidores de urease e de nitrificação [ureia (U); N-(n-butil) tiofosfórico triamida (NBPT); Dicianodiamida (DCD)]. Barras verticais indicam o erro padrão da média e valores marcados por letras iguais não diferem entre si pelo teste de Tukey, a 5\% de significância, para a peocentagem de perda acumulada de $\mathrm{N}^{-\mathrm{NH}_{3}}$ aos 21 dias após a adubação.

Mesmo o estudo ocorrendo em condições irrigadas, o que favorece a incorporação da ureia ao solo e evita perdas, as elevadas taxas de volatilização do $\mathrm{N}-\mathrm{NH}_{3}$ podem ser relacionadas com as condições climáticas durante o experimento, com temperatura máxima próxima de $40^{\circ} \mathrm{C}$, baixas taxas de umidade relativa, radiação e velocidade do vento elevadas (Figura 1). De acordo com Bremner \& Mulvaney (1978) e Sommer et al. (2004), essas condições são consideradas altamente favoráveis para volatilização de amônia.

A radiação e a velocidade do vento elevadas favorecem a maior perda de água por evaporação. A taxa de evaporação é mais importante do que a umidade inicial do solo, para determinação do momento adequado de aplicação dos fertilizantes nitrogenados sem incorporação, pois a secagem do solo potencializa as perdas de $\mathrm{N}-\mathrm{NH}_{3}$ (Lara Cabezas et al., 1992).

A redução do conteúdo de água do solo aumenta as perdas por volatilização, por causa das maiores concentrações de $\mathrm{NH}_{4}^{+}$e $\mathrm{NH}_{3}$ em solução, favorecendo a emissão da forma gasosa de $\mathrm{NH}_{3}$.

Com o aumento das doses de $\mathrm{N}$, reduziu-se a volatilização acumulada de $\mathrm{N}-\mathrm{NH}_{3}$ nas combinações de ureia e inibidores (Figura 6). Na adubação isolada com ureia, aplicada de modo localizado (em filetes), para cada grama de $\mathrm{N}$ adicionado ao solo, houve redução de $1,4 \%$
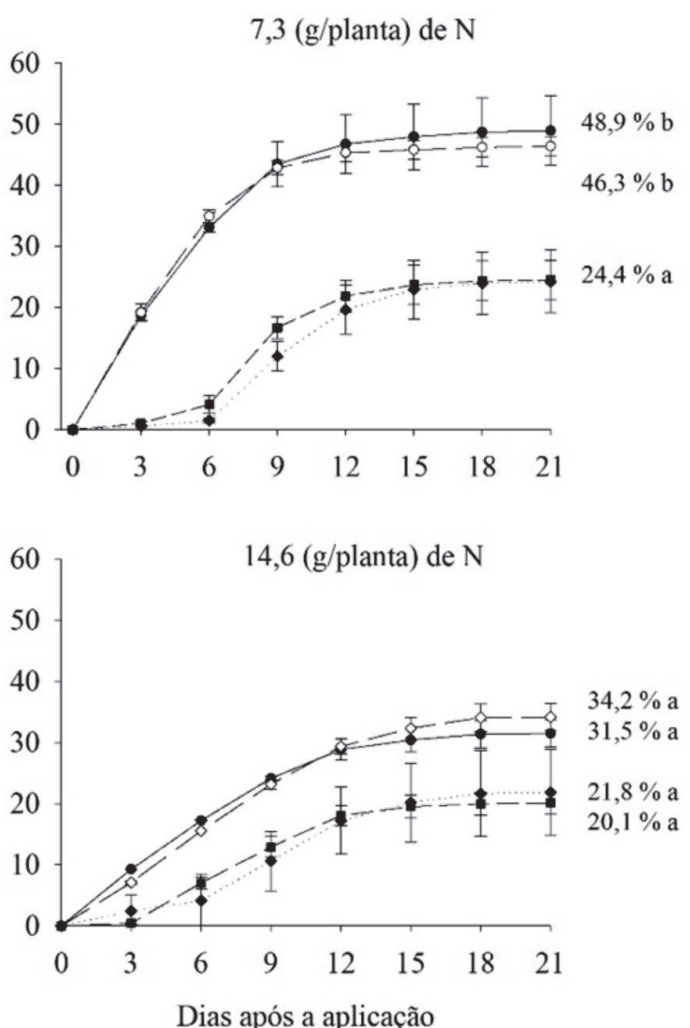


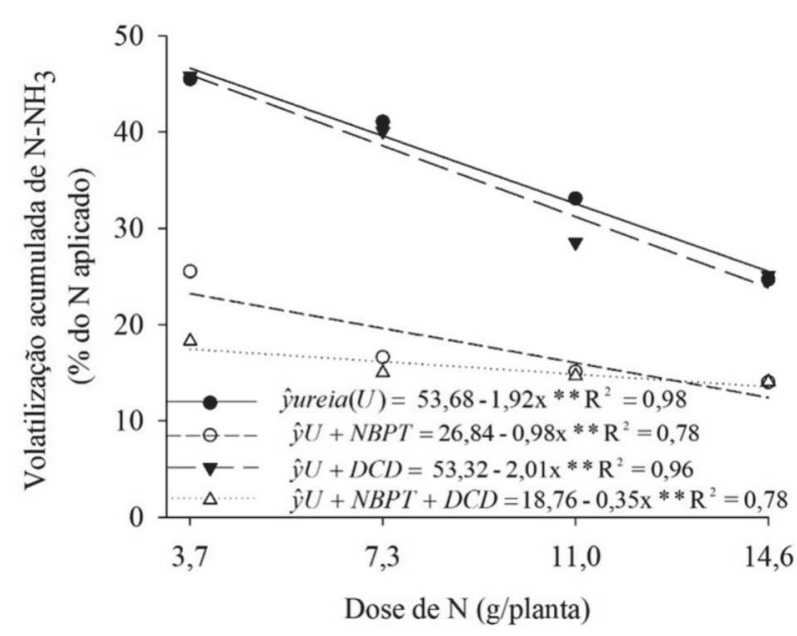

Figura 6: Perdas acumuladas de $\mathrm{N}-\mathrm{NH}_{3}$ após a adição de diferentes doses de nitrogênio e para as distintas combinações de ureia com inibidores de urease e de nitrificação [ureia (U); N-(nbutil) tiofosfórico triamida (NBPT); Dicianodiamida (DCD)]., ,*: Significativo a 5 e $1 \%$ pelo teste $\mathrm{F}$.

da volatilização de $\mathrm{N}_{-} \mathrm{NH}_{3}$; no tratamento com ureia + NBPT, a redução foi de $0,72 \%$ (Figura 6). A maior redução da volatilização, no tratamento somente com ureia, está relacionada com a maior saturação dos sítios de ação da enzima urease, pelo aumento das doses de N; enquanto, na presença de NBPT, a saturação dos sítios é ainda menor, diminuindo o efeito do aumento das doses de $\mathrm{N}$ na redução das perdas. Menores perdas de $\mathrm{N}-\mathrm{NH}_{3}$ levam à maior incorporação da molécula de ureia nos coloides do solo pela água de irrigação. Esse fenômeno aumenta o tempo para a difusão do fertilizante em maiores volumes de solo, diminuindo o potencial de perda (Cantarella, 2007).

\section{CONCLUSÕES}

As menores perdas de amônia ocorrem em baixas doses de N-ureia combinada de forma isolada com o inibidor de urease (NBPT), ou quando ambos estiverem presentes (NBPT + DCD), em condições irrigadas, no semiárido.

As condições climáticas adversas (temperaturas e radiações elevadas e umidade relativa baixa) foram os principais condicionantes para aumento da volatilização quando a ureia não recebeu o inibidor de urease.

$\mathrm{O}$ aumento das doses de nitrogênio reduz as perdas relativas de $\mathrm{N}$ por volatilização em condições irrigadas no semiárido.

\section{AGRADECIMENTOS}

Os autores agradecem à FAPEMIG (Fundação de Amparo à Pesquisa do Estado de Minas Gerais) pelo financiamento do projeto de pesquisa e, à FAPEMIG e CNPq (Conselho Nacional de Desenvolvimento Científico e Tecnológico) pela concessão de bolsas.

\section{REFERÊNCIAS}

Adfert (2015) Uremax NBPT. Disponível em: <http:// www.adfert.com.br/produtos/ uremax-nbpt/>. Acessado em: 26 de julho de 2015.

Dawar K, Zaman M, Rowarth JS, Blennehassett J \& Tumbull MH (2011) Urea hydrolysis and lateral and vertical movement in the soil: effects of urease inhibitor and irrigation. Biology and fertility of soils, 47:139-46.

Bremner JM \& Mulvaney RL (1978) Urease activity in soils. In: Burns RG (Ed.) Soil Enzymes. Londres, Academic Press. p.149-96.

Cantarella H (2007) Nitrogênio. In: Novais RF, Alvarez V VH, Barros NF, Fontes RLF, Cantarutti RB \&Neves JCL (Eds.) Fertilidade do solo. Viçosa, Sociedade Brasileira de Ciência do Solo. p.375-470.

Cantarella H, Mattos Júnior D, Quaggio JA \& Rigolin AT (2003) Fruit yield of Valencia sweet orange fertilized with different $\mathrm{N}$ sources and the loss of applied N. Nutrient Cycling in Agroecosystems, 67:215-23.

Cantarella H, Trivelin PCO, Contin TLM, Dias FLF, Rossetto R, Marcelino R, Coimbra RB \& Quaggio JA (2008) Ammonia volatilization from urease inhibitor-treated urea applied to sugarcane trash blankets. Scientia Agricola, 65:397-401.

Da Ros CO, Aita C \& Giacomini SJ (2005) Volatilização de amônia com aplicação de ureia na superfície do solo, no sistema plantio direto. Ciência Rural, 35:799-805.

Embrapa - Empresa Brasileira de Pesquisa Agropecuária (1997) Centro Nacional de Pesquisa de Solos. Manual de métodos de análise de solo. $2^{\mathrm{a} e d . ~ R i o ~ d e ~ J a n e i r o, ~ E m b r a p a . ~ 212 p . ~}$

Embrapa - Empresa Brasileira de Pesquisa Agropecuária (2013) Sistema brasileiro de classificação de solos. $3^{a}$ ed. Brasília, Embrapa. 353p.

Ferreira DF (2011) Sisvar: A computer statistical analysis system. Ciência e Agrotecnologia, 35:1039-1042.

Gioacchini P, Nastri A, Marzadori C, Giovannini C, Antisari LV \& Gessa C (2002) Influence of urease and nitrification inhibitors on $\mathrm{N}$ losses from soils fertilized with urea. Biology and Fertility of Soils, 36:129-35.

Grohs M, Marchesan E, Santos DS, Massoni PFS, Sartori GMS \& Ferreira RB (2011) Resposta do arroz irrigado ao uso de inibidor de urease em plantio convencional. Ciência e Agrotecnologia, $35: 336-45$.

Inmet - Instituto Nacional de Meteorologia (2015) Banco de Dados Meteorológicos para Ensino e Pesquisa. Disponível em:<http:/ /www.inmet.gov.br/projetos/rede/pesquisa/>. Acessado em: 26 de julho de 2015.

Lara Cabezas WAR, Trivelin PCO \& Boaretto AE (1992) Efeito do tamanho do grânulo e relação N/S da ureia aplicada em superfície na volatilização de amônia sob diferentes umidades iniciais do solo. Revista Brasileira de Ciência do Solo, 16:409-413.

Lara Cabezas WAR, Trivelin PCO, Korndorfer GH \& Pereira S (2000) Balanço nitrogenado da adubação sólida e fluida de cobertura na cultura de milho em sistema plantio direto. Revista Brasileira de Ciência do Solo, 24:363-76.

Longo RML \& Melo WJ (2005) Hidrólise da ureia em Latossolos: efeito da concentração de ureia, temperatura, $\mathrm{pH}$, armazenamento e tempo de incubação. Revista Brasileira de Ciência do Solo, 29:651-657.

Nommik H (1973) The effect of pellet size on the ammonia loss from urea applied to forest soil. Plant and Soil, 39:309-318. 
Reis MM, Santos LD, Pegoraro RF, Colen F, Rocha LM \& Ferreira GADP (2016) Nutrition of Tithonia diversifolia and attributes of the soil fertilized with biofertilizer in irrigated system. Revista Brasileira de Engenharia Agrícola e Ambiental, 20:10081013.

Sanz-Cobena A, Misselbrook TH, Arce A, Mingot JI, Diez JA \& Vallejo A (2008) An inhibitor of urease activity effectively reduces ammonia emissions from soil treated with urea under Mediterranean conditions. Agriculture, Ecosystems and Environment, 126:243-249.

Sanz-Cobena A, Misselbrook T, Camp V \& Vallejo A (2011) Effect of water addition and the urease inhibitor NBPT on the abatement of ammonia emission from surface applied urea. Atmospheric Environment, 45:1517-1524.

Soares JR, Cantarella H \& Menegale MLC (2012) Amonia volatilization losses from surface-applied urea with urease and nitrification inhibitors. Soil Biology \& Biochemistry, 52:82-89.

Sommer SG, Shjoerring JK \& Denmead OT (2004) Ammonia emission from mineral fertilizers and fertilized crops. Advances in Agronomy, 82:557-622.

Stafanato JB, Goulart RS, Zonta E, Lima E, Mazur N, Pereira CG \& Souza HN (2013) Volatilização de amônia oriunda de ureia pastilhada com micronutrientesem ambiente controlado. Revista Brasileira de Ciência do Solo, 37:726-732.

Tasca FA, Ernani PR, Rogeri DA, Gatiboni LC \& Cassol PC (2011) Volatilização de amônia do solo após a aplicação de ureia convencional ou com inibidor de urease. Revista Brasileira de Ciência do Solo, 35:493-502.
Tedesco MJ, Gianello C, Bissani CA, Bohnen H \& Volkweiss SJ (1995) Análises de solo, plantas e outros materiais. $2^{\text {a }}$ ed. Porto Alegre, UFRGS.174p. (Boletim técnico, 5).

Trenkel ME (2010) Slow and controlled-release and stabilized fertilizer: an option for enhancing nutrient efficiency in agriculture. Paris, International Fertilizer Industry Association. $160 \mathrm{p}$.

Trivelin PCO, Oliveira MW, Vitti AC, Gava GJC \& Bendassolli JÁ (2002) Perdas de nitrogênio da ureia no sistema solo-planta em dois ciclos de cana-de-açúcar. Pesquisa Agropecuária Brasileira, 37:193-201

Zaman M, Nguyen ML, Blennerhassett JD \& Quin BF (2008) Reducing $\mathrm{NH}_{3}, \mathrm{~N}_{2} \mathrm{O}$ and $\mathrm{NO}_{3}$ e $\mathrm{N}$ losses from a pasture soil with urease or nitrification inhibitor sand elemental S-amended nitrogenous fertilizers. Biology and Fertility of Soils, 44:693705 .

Zaman M, Saggar S, Blennerhassett JD \& Singh J (2009) Effect of urease and nitrification inhibitors on $\mathrm{N}$ transformation, gases emitions of ammonia and nitrous oxide, pasture yield and $\mathrm{N}$ uptake in grazed pasture system. Soil Biology and Biochemistry, $41: 1270-1280$

Zaman M \& Blennerhassett JD (2010) Effect of the different rates of urease and nitrification inhibitors on gaseous emissions of ammonia and nitrous oxide, nitrate leaching and pasture production from urine patches in an intensive grazed pasture system. Agriculture Ecosystems and Environment, 136:236246. 